\title{
Preservation of response-outcome associations through extinction
}

\author{
ROBERT A. RESCORLA \\ University of Pennsylvania, Philadelphia, Pennsylvania
}

\begin{abstract}
In four experiments, rats learned initial response-outcome (R-O) associations in an instrumental training task. They then were given training of those same responses either with another outcome (Experiments 1 and 2) or with nonreward (Experiments 3 and 4). The presence of the original $\mathrm{R}-\mathrm{O}$ associations was detected by measuring the depressive effect on responding of devaluing that $\mathrm{O}$ by pairing with $\mathrm{LiCl}$. The results indicated that the original $\mathrm{R}-\mathrm{O}$ associations remained intact through both potentially interfering treatments.
\end{abstract}

There is now compelling evidence that instrumental training can result in the formation of an association between the executed response $(R)$ and earned outcome $(0)$. Moreover, recent results suggest that once that $\mathrm{R}-\mathrm{O}$ association is formed, it is relatively impervious to various attenuating experimental manipulations. For instance, separate occurrences of the response without $O$, independently of $\mathrm{O}$, or with an alternative outcome all seem to leave the original $\mathrm{R}-\mathrm{O}$ association relatively unscathed (e.g., Rescorla, 1991, 1992). Although some of those manipulations produce marked changes in the likelihood of the response, they apparently do not do so primarily by undermining the $\mathrm{R}-\mathrm{O}$ association.

However, that conclusion is based exclusively on the use of a particular transfer procedure for the assessment of the R-O association. Colwill and Rescorla (1988) reported that a stimulus (S) that signaled the availability of a particular outcome has a specific augmenting effect on transfer responses that had earned the same outcome. Because a comparable increase is not observed in responses that have earned alternative outcomes, one may use the augmenting ability of such a stimulus to detect the presence of an association between a response and a particular outcome. In the earlier reports from this laboratory, that transfer technique has found continued presence of $\mathrm{R}-\mathrm{O}$ associations after the various operations intended to produce their elimination.

The present report describes a series of parallel experiments that assess the status of the $\mathrm{R}-\mathrm{O}$ association using an alternative technique, devaluation of the outcome. Postlearning manipulation of the current value of $O$ produces a strong change in the likelihood of a response that had earned that $\mathrm{O}$. For instance, in one early experiment, Colwill and Rescorla (1985) trained rats to make two different responses, each yielding a particular outcome, and then

This research was supported by National Science Foundation Grants BNS 83-08176 and BNS-88-03514. Correspondence concerning this article should be addressed to R. A. Rescorla, Department of Psychology, University of Pennsylvania, 3815 Walnut Street, Philadelphia, PA 19104. paired one of those outcomes with $\mathrm{LiCl}$. The $\mathrm{O}-\mathrm{LiCl}$ pairing led to selective rejection of the outcome; more importantly, the response that had previously earned that outcome was selectively reduced in likelihood. This provides relatively straightforward evidence that the organism had learned an $\mathrm{R}-\mathrm{O}$ association.

The use of this procedure to explore interactions among $\mathrm{R}-\mathrm{O}$ associations is of interest for several reasons. First, the transfer and devaluation techniques have routinely been used in this laboratory as comparable means of exposing $\mathrm{R}-\mathrm{O}$ associations. The continued ability to detect $\mathrm{R}-\mathrm{O}$ associations not only with the transfer but also with the devaluation technique would greatly strengthen the view that they have in fact survived various decremental operations. Second, because the mechanisms by which transfer exposes $\mathrm{R}-\mathrm{O}$ associations are not fully understood, transfer allows somewhat limited inferences about the status of the $\mathrm{R}-\mathrm{O}$ association. Although transfer data make it clear that the $\mathrm{R}-\mathrm{O}$ association is preserved, they do not distinguish between two important possibilities: (1) that the original $\mathrm{R}-\mathrm{O}$ association continues to participate fully in producing behavior, even after intervening treatment, or (2) that the original $\mathrm{R}-\mathrm{O}$ association becomes dormant but is selectively retrieved by the presentation of an $\mathrm{S}$ associated with the same $\mathrm{O}$. The use of the devaluation procedure as an alternative detection device can help provide additional information on these possibilities. Because there is no special retrieval cue presented during a test, sensitivity to devaluation of $O$ would suggest routine contribution of that $O$ to performance.

The four experiments reported here therefore involved a systematic replication of a sample of the earlier transfer experiments, but using devaluation as the procedure for assessing the R-O association. Experiments 1 and 2 explored the impact on an $\mathrm{R}-\mathrm{O}$ association of subsequent experience with that same $\mathrm{R}$ earning an alternative $\mathrm{O}$. Experiments 3 and 4 evaluated the effect of nonreinforced occurrences of the R. All of the experiments used standard operant procedures in which rat subjects earned outcomes such as pellets and liquid sucrose upon making responses such as leverpresses and chainpulls. 


\section{EXPERIMENT 1}

Experiment 1 examined the strength of an association between a response and an outcome as a function of whether or not that response subsequently led to a second outcome. The experimental design, modeled after that of Experiment 4 in Rescorla (1991), is shown in Figure 1.

Each animal was trained with two pairs of responses. One pair (e.g., R1 and R2) was a leverpress and a chainpull; the other pair (e.g., R3 and R4) was a nosepoke and a handlepull. Within each pair, one response was followed by $\mathrm{O} 1$ and the other was followed by $\mathrm{O} 2$. One of the pairs of responses (R1 and R2) then received additional training in which the outcomes were reversed; the other pair of responses (R3 and R4) was spared that training. All animals then received devaluation of one of the outcomes and then two choice tests, one in which R1 and R2 were the alternatives and one in which R3 and R4 were the alternatives. The choice between R3 and R4 allowed a verification of the presence of their simple associations with the outcomes. Those associations should be revealed by the preference for R4 over R3, because the latter's outcome had undergone devaluation. The choice between $\mathrm{R} 1$ and R2, however, involves two alternatives, each paired sequentially with both outcomes, but in the opposite order. The issue of interest was whether responding would be more adversely affected by devaluing the first or the second outcome that each response had earned. If subsequent training produced retroactive interference, then the second $\mathrm{R}-\mathrm{O}$ association might be dominant, and devaluing the second outcome should be more effective; but if prior training produced more proactive interference, then the first $\mathrm{R}-\mathrm{O}$ association might be dominant, and devaluing the first outcome should be more effective.

\section{Method}

\section{Subjects and Apparatus}

The subjects were 16 male Sprague-Dawley rats about 90 days old. They were housed in individual cages and maintained on a fooddeprivation regime that kept them at $80 \%$ of their ad-lib body weights. They had free access to water in the home cage.

The apparatus consisted of eight operant chambers measuring 22.9 $\times 20.3 \times 20.3 \mathrm{~cm}$, similar to those used in previous reports (e.g., Colwill \& Rescorla, 1985). The two end walls of each chamber were aluminum; the side walls and ceiling were clear Plexiglas. Each chamber had a recessed food magazine in the center of one end wall. Two small metal cups measuring $1.25 \mathrm{~cm}$ in diameter

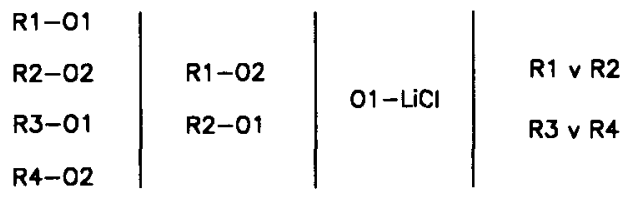

Figure 1. Experimental design for Experiment 1. Two pairs of responses (R1 and $R 2$, and $R 3$ and $R 4$ ) were trained using two different outcomes (O1 and O2). One pair of responses then received additional training with the alternative $\mathrm{Os}$. One of the outcomes was paired with the toxin $\mathrm{LiCl}$, and two choice tests were given. and $1.5 \mathrm{~cm}$ deep were sunk side by side in the floor of each food magazine. To the left of the magazine was a lever, and to the right was a chain suspended from a microswitch mounted on the lid of the chamber. Located directly above the food magazine was a $2-\mathrm{cm}$ opening behind which was an aluminum plate that activated an attached microswitch when displaced by a nosepoke. Inserted under the grid floor, just to the right of the magazine aperture, was a flat metal rod, one end of which was bent back to form a handle. An upward pull on this handle operated a microswitch. Access to these manipulanda could be blocked by covering the lever with a metal shield, retracting the chain through a hole in the ceiling, covering the nose-poke opening with a jeweled lens, and removing the handlepull from under the grid floor. The floor of the chamber was composed of $0.48-\mathrm{cm}$ stainless steel rods, spaced $1.9 \mathrm{~cm}$ apart. Each chamber was enclosed in a sound- and light-resistant shell. Mounted on the inside wall of this shell were speakers that permitted the presentation of a white noise and an $1800-\mathrm{Hz}$ tone, each measuring approximately $76 \mathrm{~dB}$ re $20 \mu \mathrm{N} / \mathrm{m}^{2}$ against a background level of $62 \mathrm{~dB}$. Also mounted on that wall was a $6-\mathrm{W}$ bulb that could be illuminated to provide a light stimulus during the otherwise dark session. The outside ceiling of the shell supported two solenoidoperated gravity feed valves that were connected via plastic tubing to the cups in the food magazine. One system permitted the presentation of $0.3 \mathrm{ml}$ of an $8 \%$ sucrose solution; the other permitted the presentation of $0.3 \mathrm{ml}$ of a $15 \%$ Polycose solution. Also attached to that food magazine was a dispenser containing 45-mg pellets (P.J. Noyes Co., Formula A).

Experimental events were controlled and recorded automatically by relays and microprocessors located in an adjoining room.

\section{Procedure}

Initial training. On the first day, the animals received two 20-min magazine training sessions, the first containing 20 pellet deliveries and the second containing 20 sucrose deliveries. Over the next 4 days, all animals were trained to make all four responses. Each training session allowed responding to earn 25 deliveries of the appropriate outcome on a continuous reinforcement schedule. Half of the animals received one session in which leverpressing led to pellets and one in which chainpulling led to sucrose; for the other half of the animals, the contingencies were reversed. In an orthogonally arranged fashion, half of the animals received one session in which the nosepoke response led to pellets and one in which the handlepull response led to sucrose; for the other half of the animals, the contingencies were reversed. Throughout this initial training, individual shaping was used if necessary for a particular $\mathrm{R}-\mathrm{O}$ combination.

First-outcome training. Over the next 10 days, the animals received VI $60-\mathrm{sec}$ training with both the leverpress and the chainpull. The $\mathrm{R}-\mathrm{O}$ associations trained were the same as those given during initial training. Each day, the animals received two 20-min sessions, one with leverpress and one with chainpull or one with nosepoke and one with handlepull. By the end of this phase, each animal had received five 20 -min sessions of VI training with each of the four responses.

Second-outcome training. On each of the next 5 days, the animals received additional VI training with one pair of manipulanda, with the outcomes interchanged, relative to the prior training. For half of the animals, this additional training was given with leverpress and chainpull; for the other half of the animals, it was given with nosepoke and floorpull.

Devaluation. Next, the animals received five 2-day cycles intended to devalue one of the outcomes. Throughout this period, all manipulanda were removed from the chambers, and one out-

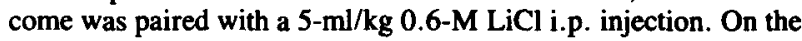
first day of each cycle, one outcome was programmed for delivery 20 times at a rate of $1 / \mathrm{min}$. At the end of the session, the animal was removed from the chamber, given the $\mathrm{LiCl}$ injection, returned 
to its home cage, and fed $2 \mathrm{~h}$ later. On the second day of each cycle, the procedure was the same with the other outcome, but no injection was given. On the fourth and fifth cycles, the doors on the isolation chambers were left open to permit observation of the animals. On those days, an animal was removed and given its injection as soon as five reinforcer deliveries were left unconsumed. The outcome devalued was selected such that for half of the animals it was pellets and for the other half it was sucrose.

Test. The next day, the animals received two 10 -min test sessions. One session offered a choice between leverpress and chainpull; the other offered nosepoke and floorpull. No outcomes were delivered during these sessions.

\section{Results and Discussion}

Response training proceeded smoothly. By the end of the first phase of training, the mean number of responses per minute was 13.6. After the second phase, it was 13.5 responses per minute. The similarity of response rates may seem surprising in light of the additional training in Phase 2. But that same pattern was observed with the same training procedures in prior experiments (Rescorla, 1991). Although there were only minor differences as a function of response identity, responses earning pellets yielded, in general, a higher rate than did those earning sucrose.

The test session data are shown in Figure 2. The leftmost pair of bars shows the results for responses that were trained on only one outcome when that outcome was subsequently devalued $(+)$ or not $(-)$. It is clear that when a response had earned only one outcome, devaluation of that outcome markedly reduced responding. Even in the context of the learning of many responses, a simple devaluation effect can be obtained. Despite this complexity in the training, the animal does not become indifferent to the details of the $\mathrm{R}-\mathrm{O}$ pairings.

The rightmost pair of bars shows the results for the responses trained first with one outcome and then with a

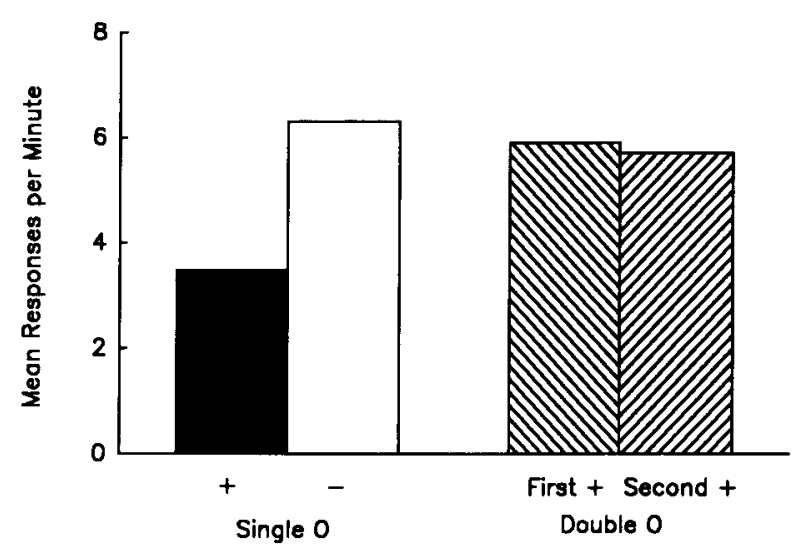

Figure 2. Responding during the test phase of Experiment 1. The left bars show the rate for the response trained with only one outcome when that outcome had been devalued $(+)$ or not $(-)$. The right bars show the rate for the response trained with two outcomes when either the first-used or the second-used outcome was paired with $\mathrm{LiCl}$. second outcome. Responding is shown separately for the case in which the first-used and the second-used outcome had been devalued. It is clear that sequential training with two outcomes resulted in a response that was not differentially sensitive to devaluation of the first and second outcome.

Because there were substantial individual differences in the initial response rates across the various responses, for statistical purposes the data from the postdevaluation test session were expressed in relation to responding prior to devaluation. For each animal and each response, a ratio of the form $A /(A+B)$ was calculated, in which $A$ is the response rate during the test and $B$ is the rate on the last day of Phase 1 treatment. The mean ratios were .17 and .30 for the single-outcome responses whose outcome had been devalued and those whose outcome had not been devalued, respectively; the comparable numbers for the double-outcome responses were .29 and .30 . The singleoutcome response with a devalued outcome was reliably below both the other single-outcome response and both double-outcome responses [Wilcoxon $T \mathrm{~s}(16)<26$, ps $<$ $.05]$. No other differences approached reliability.

The comparable levels of performance on the responses trained with both outcomes, despite devaluation of either the first or the second outcome, suggests comparable contributions of both $\mathrm{R}-\mathrm{O}$ associations. One might have anticipated that when a response first produces $\mathrm{Ol}$ and then that outcome is omitted in favor of $\mathrm{O} 2$, then the second $\mathrm{R}-\mathrm{O} 2$ association would normally control performance. The earlier failure of Rescorla (1991) to find an order effect in transfer experiments might not be inconsistent with such a view, since a transfer stimulus associated with $\mathrm{O} 1$ might be seen as a strong retrieval cue. The presence of an $\mathrm{S}$ associated with $\mathrm{O} 1$ might reveal a dormant $\mathrm{R}-\mathrm{O} 1$ association that does not normally control performance. However, with the present devaluation technique there is no strong retrieval cue to serve this function. The indifference of responding to the devaluation of the firstused or the second-used outcome therefore suggests that they both contribute to current performance.

One interpretation of the present results is that training a response sequentially with two outcomes leaves each of the R-O associations fully intact. That is, there may be neither retroactive nor proactive interference. This conclusion would agree with that drawn by Rescorla (1991) on the basis of a transfer assessment. However, an alternative account could be given in terms of equal retroactive and proactive interference. That is, training with the second $\mathrm{O}$ might interfere with the initial $\mathrm{R}-\mathrm{O} 1$ association but replace it with a functionally equivalent $\mathrm{R}-\mathrm{O} 2$ association. In that case also, one might expect equal contributions from devaluation of either outcome.

One approach to separating these alternatives is to compare single-outcome and double-outcome responses. Such comparisons are complicated by the differences in the amount of overall training experience. The additional training might well have made the double-outcome response generally stronger during the test. Nevertheless, 
there may be some information in the similarity of performance between the double-outcome responses and the single-outcome response whose outcome had not been devalued. An account in terms of equal interference might expect both of the double-outcome responses to be relatively more depressed. In the final test, whichever outcome had been devalued, the double-outcome response would have to depend on an association that had been weakened by training with another outcome. On the other hand, an account in terms of no interference more naturally anticipates comparable levels. Whichever interpretation is applied, these results suggest that the firstacquired $\mathrm{R}-\mathrm{O}$ association contributes as substantially as does the second to ongoing performance.

It is possible, of course, that the failure to find a difference between devaluation of the first outcome and the second outcome reflects an insensitivity of measurement. However, the sensitivity of the single-outcome response to devaluation suggests that response rates in the same range can readily be modulated by outcome devaluation. Moreover, the overall levels of performance in the test lie well below the predevaluation response rates and well above zero, suggesting that neither ceiling nor floor effects is responsible for the absence of differences.

\section{EXPERIMENT 2}

The results of Experiment 1 showed a surprising equivalence in sensitivity of responses trained successively with two outcomes to differential devaluation of each of those outcomes. Because of the importance of the implication that there is no effect of the order of administration of two outcomes, it seemed wise to conduct a systematic replication of this result. For this purpose, a simplified version of Experiment 1 was conducted. Each rat received instrumental training with only two responses, one of which was followed first by pellets and then by sucrose and the other of which had the outcomes given in the opposite order. One of the outcomes was then devalued, and the animal was tested separately with each of the two responses. The issue was whether responding would differ as a function of devaluation of the first-used or the secondused outcome.

\section{Method}

\section{Subjects and Apparatus}

The subjects were 16 male Sprague-Dawley rats maintained in the same manner as in Experiment 1. The apparatus was that of Experiment 1.

\section{Procedure}

Initial magazine and response training were conducted in the manner of Experiment 1. Each animal was trained to leverpress and chainpull, with the pellet and sucrose outcomes different for the two responses and counterbalanced across animals. Each animal then received 4 days of VI $60-\mathrm{sec}$ training. Each day, they received two 20-min sessions, one with leverpress and one with chainpull, with different outcomes. For the following 4 days, this VI training

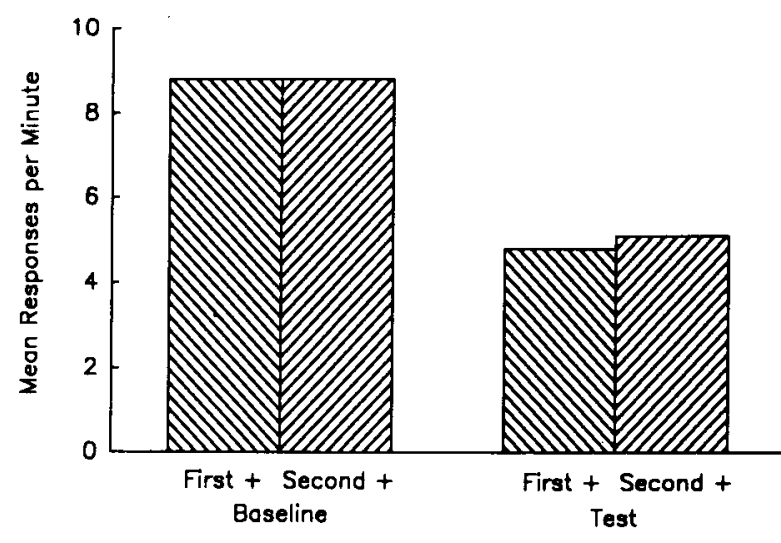

Figure 3. Responding during the final training and test phases of Experiment 2. The left bars show the rate of occurrence of responses whose first-used or second-used outcome would subsequently be devalued. The right bars show the comparable data after devaluation.

continued in the same manner, except that the outcomes that followed each manipulandum were reversed.

Over the next 8 days, all animals received four 2-day devaluation cycles in the manner of previous experiments. For half of the animals, pellets were paired with $\mathrm{LiCl}$ and sucrose was presented alone; for the other half, the outcomes were interchanged. The selection of the outcome to be devalued was orthogonal to the previously arranged $\mathrm{R}-\mathrm{O}$ relations.

The next day, all animals received two 10-min test sessions, one with each manipulandum. No outcomes were delivered during these sessions.

\section{Results and Discussion}

Initial training proceeded without incident. There were no reliable differences as a function of response or outcome identity. During devaluation, all animals came to reject the devalued outcome but continued to consume the outcome not followed by $\mathrm{LiCl}$.

The results of responding during the final training session, prior to devaluation, and the two test sessions, after devaluation, are shown in Figure 3. The data have been separated for responses whose first-earned and secondearned reinforcers had been devalued. Recall that because of the design, a response whose first-earned outcome had been devalued also had a second-earned outcome that was not devalued. It is clear from Figure 3 that, although responding was generally lower during the extinction test relative to that observed prior to devaluation, there were no differences as a function of which outcome had been devalued. The two responses showed quite similar levels during the test session $[T(15)=53, p>.20]$.

These results agree with those of Experiment 1 in finding no special sensitivity to devaluation of either the firstused or the second-used outcome. Even when only two responses are trained with each animal and when the test session involves exposure to only one response in the manner of original training, there is no evidence that the cur- 
rent value second-used outcome is more important to generating responding.

\section{EXPERIMENT 3}

As Rescorla (1991) has noted, sequentially using two different outcomes for the same response can be thought of as involving extinction of the initially learned $\mathrm{R}-\mathrm{O}$ association. During training with the second outcome, the response occurs repeatedly but the original outcome is omitted. This is one reason that one might have expected responding during the previous test sessions to be especially controlled by the value of the second outcome. However, it may be that the presence of the second outcome somehow protects the first R-O association from being undermined. Consequently, in Experiments 3 and 4 , the simple effect of nonreinforced exposure was explored directly, using the devaluation technique.

In Experiment 3, a procedure like that of Experiment 1 was employed, except that extinction replaced additional training with a second outcome. That is, each animal received training with two pairs of responses, with one member of each pair earning pellets and the other earning sucrose. One pair of responses then received extinction, but the other pair did not, prior to devaluation of one outcome. This procedure allows us to verify the continued presence of $\mathrm{R}-\mathrm{O}$ associations for nonextinguished responses and compare them with $\mathrm{R}-\mathrm{O}$ associations for extinguished responses.

It is important to note, however, that unlike training with a second outcome, extinction with one pair of responses can be expected to result in their occurring at a low level relative to the nonextinguished responses. The resulting difference in overall level of responding will complicate evaluation of the effects of devaluation. Consequently, in order to reduce this difference in overall level of responding, all responses received common training with a third outcome, Polycose, between extinction and devaluation. The results of Experiments 1 and 2 suggest that such additional training with a different outcome should not, by itself, affect the original $\mathrm{R}-\mathrm{O}$ associations; consequently, it can be used to elevate responding in order to provide more comparable levels of responding. A similar procedure, based on the same reasoning, was employed by Rescorla (1991).

\section{Method}

\section{Subjects and Apparatus}

The subjects were 16 male Sprague-Dawley rats maintained in the same manner as in Experiment 1. The apparatus was that of Experiment 1.

\section{Procedure}

The procedure was identical with that of Experiment 1 with two exceptions: (1) the second phase of training consisted of five 20-min extinction sessions with each member of one of the pair of responses and (2) this was followed by 4 days of retraining of all responses using a Polycose reinforcer. Each day, the subject received two 20-min sessions during which one response was available for earning Polycose on a VI 60-sec schedule. For 2 days, the manipulanda were leverpress and chainpull; for the other 2 days, they were nosepoke and handlepull.

\section{Results}

Initial training proceeded smoothly. By the final day, the mean responses per minute were 10.9 , with no reliable differences as a function of either manipulandum or outcome. With extinction, the response rates dropped rapidly to an average of 0.6 per minute in the final extinction session. With Polycose, responding rapidly recovered to a mean of 7.2 responses per minute on the final retraining session. During devaluation, the animals continued to eat all of the outcomes not paired with $\mathrm{LiCl}$ but consumed none of those paired with $\mathrm{LiCl}$ by the final conditioning session.

The data of most interest, from the choice test sessions, are shown in Figure 4, which displays the mean responses per minute on the manipulanda whose outcome had been devalued both for the extinguished and for the nonextinguished responses. As in Experiment 1, the substantial between-subject variability recommended that the data from the postdevaluation test session be expressed in relation to responding prior to devaluation for statistical purposes. For each response, a ratio of the form $A /(A+B)$ was calculated for each animal. In this ratio, $A$ is the response rate during the test and $B$ is the rate on the last day of retraining. The mean ratios were .19 and .42 for the extinguished response whose outcome had been devalued and for that whose outcome had not been devalued, respectively; the comparable numbers for the nonextinguished response were .34 and .49 . The lower level of responding for the manipulandum whose outcome had been devalued was reliable for both the extinguished response $[T(16)=11.5, p<.01]$ and the nonextinguished response $[T(15)=22.5, p<.05]$. The magnitude of the depression produced by devaluation was not reliably different for the extinguished and nonextinguished responses.

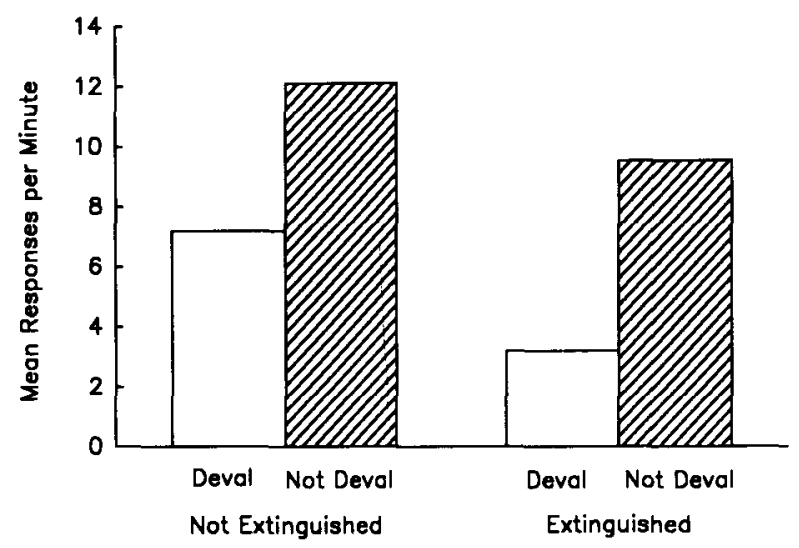

Figure 4. Responding during the test session for Experiment 3. The left pair of bars shows the rate of occurrence of responses that had been not been extinguished when their earned outcomes had or had not been devalued. The right pair of bars shows the comparable data for responses that had undergone extinction. 
However, the overall level of responding was lower for the extinguished responses $[T(16)=30, p<.05]$.

These results suggest that although extinction generally reduced the rate of responding, it did not remove the sensitivity to devaluation. Moreover, the magnitude of that sensitivity seems comparable for extinguished and nonextinguished responses.

It is of interest to contrast the results of the nonextinguished responses from the present experiment with those of the double-outcome responses in Experiments 1 and 2 . One interpretation that might be given for the failure of devaluation to yield differential results in Experiments 1 and 2 is that training with multiple outcomes makes a response generally unresponsive to devaluation. However, the nonextinguished responses of Experiment 3 were also trained with multiple outcomes and yet showed differential responding when their unique outcomes were differentially devalued, which suggests that training with more than one outcome does not in itself preclude sensitivity to devaluation.

Experiment 3 compared two responses that had each been paired with a common outcome but differed in the devaluation of their unique outcomes. Under these circumstances, differential treatment of the unique outcomes yielded differential performance, despite the presence of a shared outcome. By contrast, in Experiments 1 and 2, each response had been paired with two outcomes, and the issue was whether the response would be differentially affected by devaluation of the first or the second outcome. The equivalence of responding in Experiments 1 and 2 thus does not by itself suggest that devaluation was without impact but rather that devaluation had the same impact whether the first or the second outcome was manipulated.

\section{EXPERIMENT 4}

Experiment 4 used a variation on Experiment 2 in order to provide information on the relative strengths of extinguished and nonextinguished $\mathrm{R}-\mathrm{O}$ associations. In Experiment 3 , despite the intervening training with Polycose, the overall response rate was lower for the extinguished responses. Consequently, the results of Experiment 3 do not permit strong conclusions about whether the extinguished responses fully retained their R-O associations. Experiment 4 used the design schematized in Figure 5 to provide a stronger comparison. As in Experiment 2, each animal was trained with two responses, each initially earning one outcome and subsequently earning the alternative outcome. However, in Experiment 4, one of the responses

$$
\begin{array}{l|l|l|l|l}
\mathbf{R} 1-01 & \mathbf{R} 1-02 & 01-\mathrm{LiCl} & \mathbf{R} 1- & \mathbf{R} 1, \mathbf{R} 2 \\
2-02 & \mathbf{R 2 - 0 1} & 02-\mathrm{LiCl} &
\end{array}
$$

Figure 5. Experimental design of Experiment 4. Two responses (R1 and R2) were initially trained, using different outcomes (O1 and O2). One of those responses was then extinguished, and both were retrained using the alternative outcome. After one outcome had been paired with $\mathrm{LiCl}$, each response was presented separately in a test. received extinction intervening between its training with the two outcomes, and the other was spared extinction. One of the outcomes was then devalued. This treatment yields two responses, both trained with two outcomes; however, for one of the responses, its association with its first-trained outcome (e.g., O1) was extinguished, whereas its association with its second-trained outcome (e.g., O2) was not extinguished. This permits one to examine the effects of devaluation of either $\mathrm{O} 1$ or $\mathrm{O} 2$ on the same response. Hence, one can evaluate the effects of devaluing the outcome of an extinguished or nonextinguished association from precisely the same overall level of responding. If extinction of the $\mathrm{R}-\mathrm{O} 1$ association has a relatively specific adverse effect on that association, resulting in greater control by the $\mathrm{R}-\mathrm{O} 2$ association (which is trained in a subsequent phase), then devaluation of $\mathrm{O} 2$ should have an especially powerful effect. However, if the decrement in responding observed during extinction is accomplished by some process that is relatively nonspecific to the $\mathrm{R}-\mathrm{O} 1$ association, then one might continue to observe comparable effects of devaluing $\mathrm{O} 1$ and 02 . When the effects of this sort of extinction treatment were assessed by a transfer technique, no evidence was obtained for such a dominance (Rescorla, 1991). The present question is whether a similar conclusion will follow from the use of devaluation as the assessment technique. If so, it would strengthen the conclusion that extinction has its effects by means not specific to the original $\mathrm{R}-\mathrm{O}$ association.

\section{Method}

\section{Subjects and Apparatus}

The subjects were 16 male Sprague-Dawley rats maintained in the same manner as in Experiment 1. The apparatus was that of Experiment 1 .

\section{Procedure}

The procedure was identical to that of Experiment 2, except that all animals received extinction with one of the manipulanda between the two stages of VI training. All animals received 4 days of two 20-min VI sessions each day, one with each R-O combination. They then received 4 days of extinction with one of the manipulanda, but not the other. In each 20-min extinction session, one manipulandum was present but all outcomes were omitted. All animals then received 4 additional days of VI training with both responses, but with the outcomes reversed relative to the initial training phase. Finally, all animals received four 2-day cycles of devaluation of one of the outcomes. This was followed by a test day identical to that of Experiment 2. During the test, the importance of devaluing the first-used or second-used outcome for responses that had or had not received extinction could be assessed separately.

\section{Results and Discussion}

Initial response training proceeded as in previous experiments. At the end of the first training phase, the mean responses per minute were 8.8 . Over the course of extinction, this rate dropped to 1.1 per minute on the final extinction day. But responding recovered rapidly to a mean rate of 9.2 per minute on the fourth day of Phase 2 of training, with no residual effects of extinction on re- 


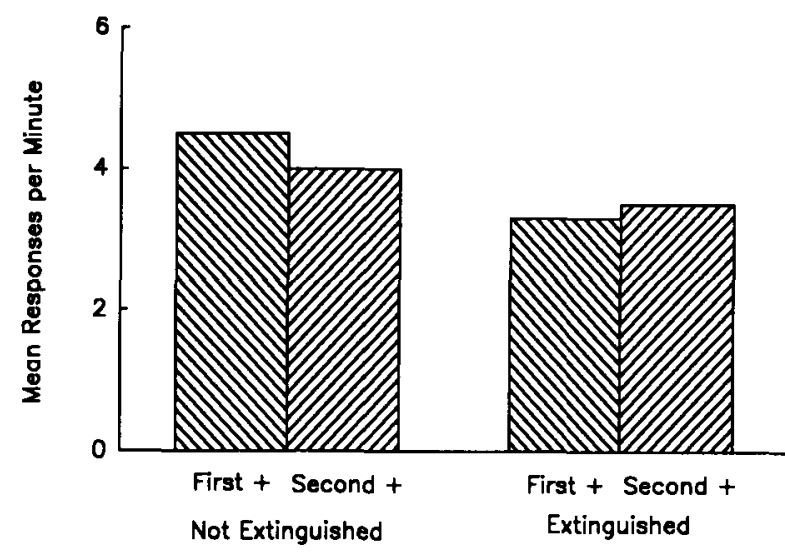

Figure 6. Results of the test session in Experiment 4. The left pair of bars shows the rate of occurrence of nonextinguished responses when their first or second earned outcome was devalued. The right pair of bars shows the comparable data for responses that had been extinguished.

sponding. Devaluation resulted in rejection of the devalued outcome but not of the alternative outcome.

The results of most interest, from the test sessions, are shown in Figure 6. These data have been combined across response and outcome identities and separated according to whether or not the response had been extinguished and according to whether its first- or second-earned outcome had been devalued. Overall, there is a slightly lower level of performance for the response with a history of extinction. Although that difference fell short of significance, it is in agreement with previous results using this same procedure (Rescorla, 1991). Most importantly, comparison within extinguished and nonextinguished responses yielded no evidence of a difference between devaluing the first or second outcome in either case [Mann-Whitney $U \mathrm{~s}(8,8)>26, p s>.20]$.

The results for the nonextinguished responses confirm those of Experiment 2, using a slightly different procedure. There was no differential effect of devaluing the two outcomes for responses that had successively earned one and then the other. The results for the extinguished responses suggest that the same conclusion applies even when extinction intervened between the two training phases. Even under circumstances in which the training based on the original $\mathrm{R}-\mathrm{O}$ relation had been deliberately extinguished, devaluation of that $O$ had the same impact as did devaluation of a different outcome used to retrain the response.

\section{GENERAL DISCUSSION}

These experiments suggest that an $\mathrm{R}-\mathrm{O}$ association remains effective even when the response is subsequently trained with a different $O$ (Experiments 1 and 2) or presented without outcome (Experiments 3 and 4). Devaluation of an outcome indicated that an $\mathrm{R}-\mathrm{O}$ association continued to contribute to performance despite these treatments.
These conclusions from devaluation experiments agree with those previously drawn from transfer experiments in which the presence of $\mathrm{R}-\mathrm{O}$ associations was assessed by the ability of a stimulus to augment a response based on the identity of its associated $\mathrm{O}$. They thus strengthen the claim that $\mathrm{R}-\mathrm{O}$ associations, once trained, are relatively impervious to modification.

The transfer and devaluation procedures each have strengths and weaknesses with regard to identifying the preservation of $\mathrm{R}-\mathrm{O}$ associations through various decremental treatments. The strength of the devaluation procedure is that it allows one to assess the contribution of various $\mathbf{R}-\mathrm{O}$ associations to current performance without presenting stimuli that may themselves affect those contributions. For instance, the results of Experiment 4 showed that $\mathrm{R}-\mathrm{O} 1$, then $\mathrm{R}-$, then $\mathrm{R}-\mathrm{O} 2$ training resulted in comparable effects of devaluing $\mathrm{O} 1$ and $\mathrm{O} 2$. This suggests that final performance is as attributable to an extinguished $\mathrm{R}-\mathrm{O} 1$ as to a nonextinguished $\mathrm{R}-\mathrm{O} 2$ association. This same inference had previously been drawn from the observation that $\mathbf{R}$ is equally augmented by a stimulus associated with $\mathrm{O} 1$ or $\mathrm{O} 2$ (Rescorla, 1991). However, it seems quite possible that transfer stimuli themselves affect which $O$ is used in generating performance to $R$, perhaps by differentially encouraging retrieval of the $\mathrm{R}-\mathrm{O} 1$ and $\mathrm{R}-\mathrm{O} 2$ associations. That is, the transfer results imply the continued presence of $\mathrm{R}-\mathrm{O} 1$ associations but do not force the conclusion that they continue to be used to generate performance in the absence of a retrieval stimulus. The results of devaluation procedures do not seem susceptible to this alternative. Hence, its identification of the presence of an $\mathrm{R}-\mathrm{O}$ association suggests that the association is routinely operative in producing performance.

However, the devaluation procedure also has at least two drawbacks. First, the execution of the devaluation treatment necessarily takes place over several days. This may provide the opportunity for associations depressed by extinction or other interfering treatments to show spontaneous recovery. By contrast, the transfer procedure allows immediate assessment of the effects of decremental operations and so avoids that possibility. Second, the devaluation procedure provides substantially less information on the associative structure of a response. One must infer the continued contribution of other outcomes by examining the responding that remains when one outcome has been devalued. The need to examine the net responding prevents one from separately assessing multiple outcomes with the same response. It also frequently forces one to make predictions about null outcomes, such as those observed in several of the experiments reported here. This can sometimes leave the results open to relatively unspecific alternative accounts, such as the possibility that when a response is trained with multiple outcomes, it becomes generally resistant to change. By contrast, the transfer technique permits independent assessment of the associations of multiple outcomes with the same response. Using this procedure, a response trained with two outcomes has been found to be elevated by the stimuli previously associated with each outcome. 
This result does not seem susceptible to the possibility that responses trained with multiple outcomes are more resistant to change.

The general conclusions reached from these experiments are in agreement with those reached by others studying multiple associative opportunities. For instance, Bouton and his collaborators (e.g., Bouton, 1991; Peck \& Bouton, 1990) have suggested that when a conditioned stimulus is trained in one context and then extinguished or followed by a different outcome in a different context, the original associations are not fully removed. Returning the animal to the appropriate retrieval context allows the display of that association. Similarly, Miller, Spear and their collaborators (e.g., Miller, Jagielo, \& Spear, 1990) have argued that the decrement in responding that can occur with the passage of time can leave associations intact, as evidenced by testing under effective retrieval cues.

The addition that the present experiments make is twofold. First, they suggest that such associations may remain intact and functional even in the absence of special retrieval operations. Second, together with the earlier transfer results, they provide more direct evidence suggesting that the original associations may remain fully intact. Taken together, these experiments provide a picture of extinction and training with alternative outcomes that suggests that originally trained $\mathrm{R}-\mathrm{O}$ associations are very resistant to decrement.

However, it is clear that despite the preservation of $\mathrm{R}-\mathrm{O}$ associations, behavior is modified by some of these techniques. Consequently, it will be necessary to look elsewhere for the behavioral modifications that occur, for instance, with extinction. Although the present experiments provide relatively little positive guidance in searching for the responsible process, they do strongly suggest that it is not specific to the associations with the outcome. One possibility that remains consistent with the data is that the nonreinforcement of a response associated with a positive outcome elicits a negative emotional response, such as frustration (Amsel, 1958; Wagner, 1966). If such frustration were relatively indifferent to the identity of the outcome, it could develop its own association with the response and hence detract from the likelihood of that response whatever the other associations encouraging its occurrence.

\section{REFERENCES}

AMSEL, A. (1958). The role of frustrative nonreward in noncontinuous reward situations. Psychological Bulletin, 55, 102-119.

Bouton, M. E. (1991). Context and retrieval in extinction and in other examples of interference in simple associative learning. In $\mathrm{L}$. W. Dachowski \& C. F. Flaherty (Eds.), Current topics in animal learning: Brain, emotion, and cognition (pp. 25-53). Hillsdale, NJ: Erlbaum.

Colwill, R. M., \& Rescorla, R. A. (1985). Post-conditioning devaluation of a reinforcer affects instrumental responding. Joumal of $E x$ perimental Psychology: Animal Behavior Processes, 11, 120-132.

Colwill, R. M., Rescorla, R. A. (1988). Associations between the discriminative stimulus and the reinforcer in instrumental learning. Joumal of Experimental Psychology: Animal Behavior Processes, 14, 155-164.

Miller, J. S., Jagielo, J. A., \& Spear, N. E. (1990). Alleviation of short-term forgetting: effects of CS- and other conditioning elements in prior cueing or as context during test. Learning \& Motivation, 21, 85-95.

Peck, C. A., Bouton, M. E. (1990). Context and performance in aversive-to-appetitive and appetitive-to-aversive transfer. Learning \& Motivation, 21, 1-31.

RESCORLA, R. A. (1991). Associations of multiple outcomes with an instrumental response. Journal of Experimental Psychology: Animal Behavior Processes, 17, 465-474.

Rescorla, R. A. (1992). Response-independent outcome presentation can leave instrumental R-O associations intact. Animal Learning \& Behavior, 20, 104-111.

W AGNER, A. R. (1966). Frustration and punishment. In R. N. Haber (Ed.), Current research in motivation (pp. 229-239). New York: Holt, Rinehart and Winston.

(Manuscript received July 10, 1992; revision accepted for publication December 1, 1992). 